Meta

Journal des tradlucteurs

Translators' Journal

\title{
Travail et vocabulaire spécialisés : prolégomènes à une socio-terminologie
}

\section{Yves Gambier}

Volume 36, numéro 1, mars 1991

La terminologie dans le monde : orientations et recherches

URI : https://id.erudit.org/iderudit/002795ar

Aller au sommaire du numéro

Éditeur(s)

Les Presses de l'Université de Montréal

ISSN

0026-0452 (imprimé)

Découvrir la revue

Citer cet article

Gambier, Y. (1991). Travail et vocabulaire spécialisés : prolégomènes à une socio-terminologie. Meta, 36(1), 8-15. d'utilisation que vous pouvez consulter en ligne. 


\section{TRAVAIL ET, VOCABULAIRE SPÉCIALISÉS: PROLÉGOMÈNES À UNE SOCIO-TERMINOLOGIE}

YVES GAMBIER

Université de Turku, Turku, Finlande

Les fragments qui suivent sont un plaidoyer pour une socio-terminologie, urgente à définir devant la formalisation liée à l'informatisation et devant l'idéalisme de la théorie. La problématique est complexe: la terminologie doit accompagner une demande sinon elle risque de tomber dans une utopie fantastique, de rejoindre la comète des langues inventées; bâties sur une impeccable rationalité ou prises dans les délires de l'imaginaire. Elle ne peut être «machine à rêver» où verser fantasmes et nostalgie pour une langue ésotérique, policée, totalitaire. Elle ne peut être non plus «machine à jouer» sans objet référentiel ni sujets sociaux.

Par ailleurs l'émergence d'incertitudes - est-il possible de faire face à Babel? oblige à s'interroger: la terminologie comme théorie, comme profession, piétine car elle connaît une crise de croissance (reflet du développement des techniques, diffusion rapide des savoirs), une crise d'identité et d'autorité (reflet de la crise des instances normatives, des réductions budgétaires pour des tâches et des postes de terminologues), une crise de méthodes (méthodologie éclatée par suite de l'expansion de l'informatique qui permet des stratégies de traitement, de stockage adaptés à des besoins précis). Ces crises ne sauraient être analysées de la même manière et conduire à des conclusions identiques. En fait se pose ici la question cruciale du partage entre les terminologies comme ensemble des termes d'un domaine d'activité, fonctionnant en milieu professionnel et les terminologies issues d'un effort de normalisation, supposé régir entièrement l'usage du chercheur, du technicien. En mêlant terminologie officielle et terminologie non contrôlée, on ne peut guère réussir à valider l'action terminologique, la régulation néologique... La dimension sociale est non seulement une composante des technolectes, mais elle devrait être aussi part intégrante de la théorie terminologique.

Une terminologie dynamique ne suffit pas pour régler ces crises: l'accumulation des vocabulaires/glossaires/lexiques... facilitée par l'ordinateur, ne peut aider la terminologie à se (re)mettre sur ses pieds - pour entre autres répondre à la forte croissance des échanges scientifiques, des savoir-faire techniques et dépasser le faible rendement des banques de termes, des dictionnaires spécialisés.

La linguistique structurale, braquée sur la «langue» de locuteurs fictifs, a fait éclater son idéalisme en abordant les usages, les variations - en se faisant linguistique sociale (sociolinguistique, pragmalinguistique). L'analyse des emplois réels devrait permettre aussi de dévoiler l'idéologie qui a marqué jusqu'à aujourd'hui la terminologie; tenter de réintroduire celle-ci dans la pratique sociale qu'est tout discours, c'est tenter du même coup de comprendre les divisions des connaissances, la synonymie, les degrés d'équivalences entre langues, les emprunts..., c'est quitter les principes qui occultent les rapports de force dans la communication, les enjeux de toute énonciation, c'est quitter la définition du sujet comme seule source de concepts... 
Le caractère de la théorie a été fondé davantage sur des prescriptions que sur des descriptions - d'où ses postulats sur la monosémie, la biunivocité des termes idéals, d'où ses propositions normatives/typologiques qui ne parviennent pas à expliquer la profusion néologique, qui ne prennent pas en compte que la langue avec ses contraintes, son organisation structurale propre, constitue un «déjà-là», un paramètre parmi d'autres dans la communication.

Je distingue trois niveaux où la dimension sociolinguistique féconde la terminologie :

1) dans le travail terminographique lui-même;

2) dans la terminologie à visée normalisatrice, plus souvent en aval qu'en amont de pratiques qu'on cherche à modifier (vision réductrice des usages);

3) dans la terminologie issue de l'observation des pratiques langagières, en amont de toute intervention contrôlée.

J'aborderai ici uniquement le point 3, les deux autres ayant été traités lors d'un séminaire «Langues de spécialité et théorie de la traduction» (11-12 février 1989, VöyriFinlande, à par.), la cohérence de la problématique se dégageant de ces 3 niveaux complémentaires.

\section{I- DIMENSION SOCIOLINGUISTIQUE DE LA CREATION ET DE LA CIRCULATION TERMINOLOGIQUES «SPONTANÉES»}

L'usage spontané est posé par opposition à celui officiel $\left(2^{\mathrm{e}}\right.$ niveau $)$ : tous les deux sont contraints, c.-à-d. soumis, à des déterminations. Mais contrairement au dispositif institutionnalisé, la production et la diffusion de la masse des termes restent «incontrôlées», c.-à-d. réglées par des actes et des acteurs en contradiction. La socioterminologie à constituer parvient ici à un autre niveau d'analyse pour tenter de comprendre les rapports entre dénominations (productions langagières) et besoins conceptuels (pratiques sociales), entre travail (forces productives) et savoir/savoir-faire (dynamique cognitive).

L'émergence des termes dans les interactions de scientifiques, de techniciens, d'industriels ne se confond pas avec leur genèse étymologique, leur développement formel. Les domaines de connaissance et d'activité ont d'autres finalités qu'eux-mêmes. Ainsi, il ne semble pas possible de saisir la terminologisation d'un de ces domaines en la réduisant au seul processus cognitif (les notions appellent des dénominations); y concourent également les processus socio-professionnels. C'est donc à une socio-genèse des termes, dans l'interaction des occupations quotidiennes, que doit s'attacher la terminologie.

Le langage technico-scientifique est un faire-connaître qui vise une transposition explicative du sens et suppose un méta-discours - d'où ici les apports possibles de la sémiotique narrative à l'analyse des discours spécialisés. Parce qu'elle a toujours lieu dans une communication, la polysémie est forcément une dimension de la terminologisation (cf. par ex. Guilbert sur la naissance du vocabulaire de l'aviation 1965). La stratégie dialogique implique la dérive au moins partielle du sens: or, la rigueur théorique de la terminologie voudrait imposer et des termes monosémiques et une monosémie clairement délimitée (univocité du concept): cf. par ex. Y. Gentilhomme (1982). Cet absolu d'un contenu strictement déterminé par sa définition et rien qu'elle (obéissant aux impératifs logiques) ne tient pas, même à l'intérieur d'une discipline donnée: il peut être un postulat de la théorie, non un résultat de l'analyse. La métaphorisation, le transfert d'un terme au statut de «mot» et inversement, le passage d'un terme de tel domaine à tel autre sont des exemples fréquents de la formulation de la pensée savante, technique: au terme figé dans sa définition doit être substitué alors le terme dans son fonctionnement, comme outil dans la dynamique collective de la 
conceptualisation - outil économique puisqu'on recourt aux signes déjà dans la langue (cf. Gambier 1985). Ce glissement, issu de l'analogie sémantique, les transformations de sens, les catégorisations lexicales qui ne répondent pas à l'«objectivité» de la tradition rationaliste (Bloomfield, Chomsky) éclairent les limites de la théorie: à trop vouloir travailler sur des unités isolées, à trop vouloir séparer les domaines, à trop considérer les termes en soi, hors de toute énonciation - comme si la notion de «vocabulaire» se définissait sans rapport à l'activité discursive d'un groupe donné - elle occulte l'interactivité qui est à la base de la créativité scientifique, de l'innovation technique. Cette interactivité entraîne de nouvelles associations de sens et de termes. Toute terminologie introduit, ravive un système de valeurs (idéologiques, philosophiques, psychologiques). Cela est d'autant plus vrai que la coupure conventionnelle entre le mode d'élaboration des notions et leur mode de diffusion est de plus en plus défiée aujourd'hui par l'accélération des processus de divulgation et d'intégration des connaissances: la culture technico-scientifique ne cesse de participer davantage aux mythes de la (post)modernité.

Sans prétendre définir les «lois» qui président à la production des concepts, mais sans non plus ignorer le poids des facteurs sociaux, sémiotiques, psycholinguistiques qui orientent le développement terminologique, on ne peut plus se contenter de la définition dominante des LSP (langues de spécialité, technolectes) comme jargons, comme moyens exclusifs/emblématiques de communication interne à un groupe fermé de spécialistes. Pour dépasser cet aspect de «langue secrète», signe de ralliement à une communauté de pairs, à un pouvoir, il faut considérer la problématique de l'énoncé dans le champ historique et social des sciences et techniques, afin de pouvoir évaluer les problèmes de médiatisation - aucune connaissance ni pratique ne ressort à l'hermétisme. Un énoncé qui transmet un concept a au moins deux enjeux :

- celui de l'évolution des concepts: passer d'un mode de conceptualisation à un autre, dans un même domaine (par ex. en biologie - F. Gail : Stengers 1987);

- celui symbolique: tout concept est opérateur socio-symbolique à deux faces; il s'agit d'une part d'attirer l'intérêt/l'attention, d'autre part de faire fructifier l'intérêt d'un concept (Stengers 1987, 1989).

Une des visées de la socio-terminologie revient donc à modéliser cette double médiatisation: du concept à l'énoncé et les modes de transformation du concept et de l'énoncé, dans et hors du champ spécialisé. Il sera alors possible de traiter les termes par rapport à un système (adéquation de la désignation, rattachement à un réseau conceptuel) et dans leur fonctionnement, sur le terrain des contradictions sociales (qui utilise quoi? qui innove? comment et par qui il y a diffusion? comment s'opèrent les réajustements terminologiques, etc.). La socio-terminologie est donc cet effort de comprendre le rôle cognitif du terme chez le sujet connaissant, rôle solidaire de l'enracinement social de ce même terme et de ce même sujet - l'activité cognitive étant toujours en prise avec «l'action sur les choses c.-à-d. avec le travail nécessaire à la subsistance des hommes et à la production des objets» (Guilbert 1976: 205-206).

Dans ces conditions, information et terme deviennent des notions relatives et cessent d'être des entités en soi/pour soi, objectivement délimitées: leur contraintes de production interactive sont telles que les dénominations ne sont plus à sens unique par lequel un agent actif s'impose à un autre passif - sans conscience, sans connaissances antérieures, sans propres besoins, sans motivations... Cette négociation peut être déjà étudiée dans le cadre de l'analyse conversationnelle (voir plus loin), à défaut d'une théorie psycho-sociale du langage, souhaitée dès 1980 par Guespin (p. 129).

Les situations d'interaction sont nombreuses et complexes, dans la division sociale du travail. Plutôt que de voir les terminologies fonctionnant en vase clos (groupes de 
pairs), on est amené à considérer leur dissémination et ainsi la contradiction qui traverse toute science, toute technique : celles-ci n'existent pas sans leur diffusion, il n'y a pas de discours de spécialité sans préoccupation de propagation (du laboratoire à l'entreprise, du service à la clientèle, du commerce aux consommateurs...). À l'extension de l'usage des LSP qui ne relève pas d'un bon vouloir démocratique répond la normalisation: comme d'autres marchés, le marché terminologique connaît des tensions entre laisser-faire et dirigisme. Cette oscillation éclaire par ex. le statut de la terminologie en France: au moment où le pouvoir dominant tente de reconquérir le marché linguistique intérieur (emploi du français dans l'enseignement et la recherche 1976, loi linguistique de 1975 , Commissions de terminologie depuis 1973, choix des langues dans les colloquescirculaires de 1972 et de 1981, contre le franglais dans les années 60...), il y a crise des instances normatives, hier hégémoniques, aujourd'hui balkanisées et concurrencées par les médias... Cette oscillation explique en partie aussi les difficultés de coopération terminologique avec par ex. le Québec à la glottopolitique plutôt dirigiste fondée sur une conscience linguistique plus aiguë et sur une demande sociale différente.

Quels sont les acteurs qui jouent à l'heure actuelle un rôle terminologique? Quelle est leur idéologie, par ex. vis-à-vis de l'anglais? Comment se manifestent les légitimités terminologiques, quand les forces en présence sont antagonistes? Comment les représentations des chercheurs chez les techniciens influent-elles sur leur comportement langagier?

De telles questions n'ont pas de réponses faciles. Tout travailleur, au sens large, se trouve dans des situations d'exposition à des productions langagières en nombre, fréquence et intensité variables; ainsi certains métiers appellent à une prise de parole (orale: par ex. le chef d'atelier, le juriste... et/ou écrite: par ex. le responsable de laboratoire), leur discours étant «garanti» par l'institution, la hiérarchie...; d'autres métiers exposent à la parole instrumentale c.-à-d. que la profession s'exerce via l'autorité verbale (par ex. le chef d'équipe, le médecin, le manager, le commerçant...); d'autres enfin ne rendent pas la parole nécessaire (par ex. chez les salariés manuels, les employés de bureau...). On peut s'attendre à ce que les familiers de l'interaction (professionnellement, socialement) sauvent mieux la face en situation asymétrique d'entretien, d'interview car plus aptes à s'auto-corriger, à se reprendre, à hésiter... Ces conduites langagières - diverses donc suivant les activités socioprofessionnelles, les rangs hiérarchiques - sont à considérer évidemment dans toute approche de la manipulation et de la créativité terminologique - d'autant plus que les tâches ellesmêmes ont de plus en plus à faire avec les activités dites d'information, avec l'habilitation à parler... Aux fonctions cloisonnées de l'entreprise d'hier a succédé un ordre qui mobilise les compétences et les savoirs par une intense communication interne, ordre consensuel qui prétend à l'harmonie dans le travail entre les aspirations des financiers, des employés, des gestionnaires, des décideurs...

Il est impensable de modéliser la production et la diffusion des termes en ignorant ces perceptions et ces réalités du milieu de travail; la théorie terminologique ne peut s'en tenir aux définitions de concepts, à la pertinence des dénominations en ignorant les modes et l'importance de la catégorisation, dans l'interprétation des énoncés interactifs: elle doit ajouter aux apports de la sociolinguistique et de la sociologie de la communication (cf. $2^{\mathbf{e}}$ niveau) les acquis de l'ethnométhodologie, de la sémantique cognitive (concepts de système de catégorisation, d'implicite social, de prototypes sémantiques...) et ceux, surtout méthodologiques, de l'ethnographie de la parole, de l'analyse conversationnelle (cf. travail pionnier de Heller 1982). Ainsi, réaliser des interviews sur le processus de terminologisation présuppose une certaine typologie des métiers, des postes, des grades en fonction des degrés de nécessité de la parole, de la 
fréquence des interactions (de leur réseaux), de l'importance de l'accès aux technologies nouvelles (différences entre entreprises selon le secteur d'activité?). Cela présuppose la maîtrise conceptuelle de la dynamique de groupe, des structures de sociabilité (Guespin 1985) dans la mesure où chacun est exposé à d'autres éléments de l'intertexte que ceux connus lors de la formation initiale (via par ex. les associations professionnelles, les syndicats...). On s'éloigne alors des enquêtes descriptives, ponctuelles réalisées sur la circulation des termes officiels ( $2^{\mathrm{e}}$ niveau). Une telle approche ethnographique servirait également à mieux identifier les différences entre langue dite générale et langue dite de spécialité, où s'effacent en apparence les traits de la négociation du sens au profit de l'impersonnalité; elle servirait à approfondir la pertinence des différences données plutôt intuitivement en terminologie entre sciences exactes, sciences sociales et industries.

La complexité des paramètres à considérer explique sans doute la rareté encore des études interactionnelles dans le travail tandis que certaines interactions assez ritualisées ont été fréquemment analysées (entre médecin et patient, thérapeute et malade, juriste et prévenu...). Elle explique certainement aussi pourquoi les relevés terminographiques se sont surtout appuyés sur des sources écrites (décontextualisées) — cf. 1 er niveau.

\section{II- VERS UN MODÈLE DE LA CIRCULATION TERMINOLOGIQUE?}

Peut-on finalement envisager de modéliser à la fois la diffusion (connaissances passives) et l'implantation (usages réels) des terminologies, en intégrant les acquis, les expériences de terminologisation officielle, de changement linguistique concerté ( $2^{\mathrm{e}}$ niveau) et les résultats, encore très partiels, des analyses de terminologisation spontanée ( $3^{\mathrm{e}}$ niveau)? Un tel modèle peut-il s'appliquer de la même manière aux entreprises industrielles, dans leurs communications internes et externes, aux centres de recherche de l'enseignement (supérieur, professionnel) - une des formes de la divulgation, sinon de la vulgarisation, des termes?

Bien des grandes firmes ont un service linguistique (rédaction, documentation, traduction, terminologie...) - ex. Siemens, Philips, Aérospatiales..., comme d'ailleurs les organismes internationaux (ONU, OTAN, CEE, Banque mondiale...): cela ne prouve pas la mesure des emplois quotidiens et n'indique pas non plus les sources, les conditions d'émergence de la plupart des termes.

Ex. de paramètres dans le processus de terminologisation (sans préjuger de leurs rapports, de leur poids respectif):

\section{Caractère de l'entreprise}

- secteur d'activité/type d'industrie

- taille et localisation

- type de division du travail

- réseaux de communication

interne et externe

- fréquence des interactions

- types de parole (garantie par le rang, instrumentale, non nécessaire...)

- impact des nouvelles technologies (sur la production, les échanges internes, la formation, les qualifications...)...

\section{Caractères des médiateurs}

- leur fonction dans la hiérarchie/poste occupé

- degré de pouvoir (de décision, d'initiative...)

- formation d'origine

- spécialisation/qualifications 
- âge; lieu d'habitation

- conditions/fréquence d'accès à la formation...

\section{Usages et compétences linguistiques}

- parler/écrire/lire

- compétences objectives et perçues/auto-évaluées en langues (L1, L2)

- domaines/occasions d'emploi des terminologies

- motivations/intérêts pour le recours à une LSP...

Dans le domaine de la recherche, dans les centres forcément producteurs de concepts, de termes, comment préciser les facteurs interactifs de la terminologisation, par ex. lors d'une conférence? La science est pratique matérielle, institutionnelle et discours: ceux-ci reflètent-ils homologiquement celles-là? Quelques remarques s'imposent ici :

- la logique de la recherche, contrainte par son objet, diffère de la stratégie de son exposé contrainte par des conventions (académiques, éditoriales);

- la fabrication de la science ne va pas sans préoccupation de sa diffusion (cf. plus haut sur la médiatisation): il n'y a pas de connaissances sans énoncé orienté vers les pairs (discours légitimant, polémique) et les autres, non spécialistes du domaine (discours didactique, de vulgarisation). Ces deux genres sont en continuité pour servir le besoin de reconnaissance, de crédit..., contrairement à l'opinion courante qui les sépare en catégories étanches, sous prétexte d'une science pure, objective et d'une propagation toujours aux odeurs plus ou moins de soufre, liée à la réussite socio-économique d'un domaine (ex. l'automobile, l'astronautique), à l'occurrence d'événements forts de l'actualité (ex. l'alunissage, les accidents nucléaires). Dans un cas, la théorisation plus polémique suscite néologismes (droit et devoir du spécialiste de néologir - Y. Gentilhomme - de choisir un terme nouveau, d'agir terminologiquement), dans l'autre la créativité terminologique se banalise — R. Galisson - . La banalisation implique la néologisation, comme celle-ci réfère à l'intertexte des énoncés du même champ; les deux marquent des temps forts de la diffusion des savoirs: en retracer le mouvement, en rendre compte semble primordial pour comprendre la circulation terminologique, avec ses enjeux, ses réajustements ses reformulations - rôle par ex. de groupes-charnières dans la vulgarisation (hypothèse du $3^{e}$ homme entre le savant et le grand public). La socioterminologie, dans le domaine des sciences, ne s'appuierait pas uniquement sur l'intertextualité d'une discipline donnée mais aussi sur le continuum de la sociodiffusion: ainsi un terme connaîtrait une période de lancement puis une phase d'extension de son emploi, enfin un temps d'éclatement de la notion (polysémisation). Une telle archéologie reste à constituer - de même il reste à confirmer que le nomadisme des concepts et la problématique des termes sont plus aigus en sciences sociales qu'en sciences exactes et naturelles. En tout cas, une histoire de ces sciences est inséparable d'une terminologie diachronique - encore peu développée mais dont on peut prévoir la pertinence pour l'historien attaché aux transformations des connaissances, aux ruptures épistémologiques... Miroir d'une époque, miroir d'une stratification sociale (cf. notions de degré de lexicalisation, de néologie et de niveau de langue en LSP), les vocabulaires tendent aussi à être miroir de l'évolution des sciences et des techniques qu'on songe par ex, aux vocables de la chimie, du pétrole, de la métallurgie, de la physique...

Cette dimension historique des terminologies n'éloigne qu'en apparence de l'interrogation d'une socio-terminologie: ne peut-elle en effet permettre de répondre à la question de savoir si la terminologie aide - et jusqu'où - ou pas au changement linguistique en général, si elle concourt à l'optimisation des interactions ou si au contraire la technicisation des nomenclatures est une des formes de sélection, de sclérose, plus apte 
à la reproduction des communautés de pairs qu'à la diffusion des savoirs et des savoirfaire?

Dernier point suscité par l'essai de définition d'une socio-terminologie: quels rapports peut-elle entretenir avec la réflexion glottopolitique? Il ne suffit pas d'affirmer le besoin d'intervention des linguistes/des langagiers devant les formes nouvelles des processus d'information et de transmission (industries de la langue, banques de données, bases de connaissances en IA, TAO...). Encore faudrait-il pouvoir situer ces interventions sur le marché des langues (avec ou sans aménagement particulier), dans la rivalité des langues à prétention internationales, dans le cadre de l'internationalisation des échanges commerciaux, des programmes scientifiques, suivant les besoins des forces sociales dans la redéfinition des tâches industrielles... Le concept de glottopolitique (cf. Langages, 83, coordonné par JB Marcellesi, septembre 1986; Guespin, 1985) devrait donner un éclairage différent aux recherches en terminologie (problèmes des normes, des besoins linguistiques, du recueil/de la gestion/de la connaissance des pratiques langagières dans les structures sociales, les rapports de production): la socio-terminologie est un effort pour intégrer langue et pratiques, ces dernières étant en aval et en amont de la première, la subissant et l'«in-formant» à la fois. Dès lors, la terminologie cesserait de servir nécessairement le dirigisme linguistique (trop souvent synonyme de politique de la langue) et l'insécurité que crée toute prescription d'en haut. Une telle perception de la dynamique, du changement linguistique ne serait pas sans influer sur les programmes d'aménagement terminologique.

Nombre de propositions et d'affirmations qui précèdent peuvent paraître simplistes, dogmatiques même étant donné qu'aucun matériau, qu'aucune preuve, qu'aucune analyse de cas ne viennent les étayer. L'objectif était autre: tenter de définir les niveaux de pertinence, d'intervention d'une socio-terminologie afin de dépasser les blocages, l'idéalisme que révèlent la plupart des textes en terminologie, afin de ne pas tomber dans une vision mécaniste sur les rapports entre activité - dont le travail - et terminologie, risque couru par toute étude empirique qui ne s'interrogerait pas au préalable sur les limites et les possibilités de dépassement de la théorie dans son état actuel.

\section{BIBLIOGRAPHIE}

GAMBIER, Y. (1985): «Terminologie et métaphore», Erikoiskielet ja käännösteoria ( $5^{\mathrm{e}}$ séminaire VAKKI sur les LSP et la théorie de la traduction), 26-27 janvier 1985, Vöyri, Finlande, pp. 31-45.

GAUDIN, F. (1988): «Terminologie et sociolinguistique» (exposé à la rencontre universitaire Rouen-Hanovre, mai 1988).

GAUDIN, F. (1988): «Terminologie et travail» (exposé à la rencontre de Rouen des 17-18 octobre 1988: Linguistique et matérialisme).

GENTILHOMME, Y. (1982): «De la notion de notion à la notion de concept. Processus itératif d'acquisition des notions», Travaux du Centre de Recherches sémiologiques, 42-1, juillet 1982, pp. 63-107.

GUESPIN, L. (1980) : «Langage et travail, de l'anthropologie à la théorie de la personnalité», La Pensée, 209 , janvier 1980, pp. 114-129.

GUESPIN, L. (1985): «Les structures de la sociabilite, un niveau d'analyse pertinent pour l'approche linguistique», La Pensée, 244, mars/avril 1985, pp. 120-130.

GUESPIN, L. (1985): «Introduction. Matériaux pour une glottopolitique», Cahiers de linguistique sociale, 7 , éd. A. Winther (Actes du Colloque: Problèmes de Glottopolitique, Mont Saint-Aignan, 20-23 septembre 1984), pp. 13-32.

GUILBERT, L. (1976): «Terminologie et linguistique», Actes du Colloque Essai de définition de la terminologie (Québec-OLF), repris dans: Rondeau, Felber: 1981/ Textes choisis en terminologie, GIRSTERM/Laval, Québec, pp. 199-120.

HELLER, M. et al. (1982): «Le processus de francisation dans une entreprise montréalaise: unc analyse sociolinguistique», OLF-Coll. Langues et Sociétés, 203 p.

SLOTT, H. (1984): Finns det en terminologisk medvetenhet $i$ samhället $i$ dag? (Y a-t-il une conscience terminologique dans la société d'aujourd'hui?), synthèse d'un colloque de Nordterm, Stockholm,.28-29 mai 
1984, Tekniska Nomenklaturcentralen, 49 p. (sensibilisation des producteurs de termes et des usagers à la Cie norvégienne de pétrole, chez Ericsson en Suède, à l'Autorité maritime de Finlande, etc.). STENGERS, I. (ed.) (1987): D'une science à l'autre, des concepts nomades, Le Seuil, $393 \mathrm{p}$.

STENGERS, I. et J. SCHLANGER (1989): Les concepts scientifiques : invention et pouvoir, La Découverte, coll. Anthropologie des sciences.

TOURANGEAU, R. (1982): «Pour une contribution sémiotique de la traduction et de la terminologie», Meta, 35-3, pp. 310-314. 